ISSN 1823-626X

Journal of Fundamental Sciences

available online at http://jfs.ibnusina.utm.my

\title{
A feedback queue with additional optional batch service
}

R. Kalayanaraman and S. Sumathy

Department of Mathematics, Annamalai University, Annamalai Nagar, India.

Received 5 June 2010, Revised 30 August 2010, Accepted 7 September 2010, Available online 1 November 2010

\begin{abstract}
A single server infinite capacity queuing system with Poisson arrival process along with Bernoulli feedback decision process is considered wherein the server provides two types of service. The first essential service is rendered one by one to all the customers and second optional service is given in batches of fixed size b. For this model the steady state probability generating function for the queue length process has been obtained and average queue length has been found explicitly. Results for particular cases are obtained and some numerical results are presented to test the feasibility of the queuing model.
\end{abstract}

| Bernoulli process | Queue length distribution | Feedback |

( 2010 Ibnu Sina Institute. All rights reserved. http://dx.doi.org/10.11113/mjfas.v6n2.194

\section{INTRODUCTION}

Consider a queueing system in which a customer having received service, may return to the waiting line under some decision rule to receive another service, these services are called essential services. In addition the customer may require additional service of different type called optional service. Such a queueing system is called feed back queueing system with additional optional service.

Many practical queuing systems especially those with feedback have been widely applied to real life situations, such as the problem involving hospital emergency wards handling critical patients, and unsatisfied customers in public telephone booth of coin box type etc. The formulation of queue with feedback mechanism has been given by Takacs (1963). Such queuing models have been studied by various authors including D’Avignon et al. (1976), Kleinrock (1975), and Kalyanaraman et al. (2002).

The present paper deals with a simple $\mathrm{M} / \mathrm{M} / 1$ feedback queuing system wherein the server, apart from providing the essential service to all the customers, also provides an additional optional service to the customers in batches of fixed size $b(\geq 1)$. The idea of optional service is quite relevant to many situations such as, among the passengers who travel to a big town by airlines, trains, ships, etc. only a part of them take interest to travel to an interior destination of tourist importance. This type of queue has been dealt with by several researchers notable among them is Madan $(1975,1992)$.

Corresponding author at: Department of Mathematics,

E-mail address: r.kalyan24@rediff.com
The rest of the paper is organized as follows. The model studied is described in section 2 with a diagrammatic representation. In section 3 we derive the probability generating function of the steady state probabilities for this model. In section 4 we obtain the closed form expressions for the mean queue and average waiting time. Some particular cases are deduced in section 5 and a numerical study is presented in section 6 . The contribution of the paper is given in the conclusion section.

\section{The Model}

The model we consider here is a single server queue with Poisson arrival process whose mean value is $\lambda \mathrm{t}$. Each arrival is given a first essential service, whose service time is exponential with mean $1 / \mu_{1}$; after completion of the first essential service the customer leaves the system with probability q or he may demand additional service called optional service with probability p. In the case of optional service the customers are first asked to queue up and as soon as there is a batch of size is b, ready for optional service, the server suspends the first service irrespective of number of customers in the main queue and starts the optional service whose service time is also exponential with mean $1 / \mu_{2}$. As an additional assumption, if there is no waiting customer in the main queue, a batch of size less than b can be taken for optional service. Sometimes, the customers may need individual attention of the server, who are not willing to get a batch service, and in this case the customer may feedback into the head of the main queue with probability $r$, where $p+q+r=1$. The diagrammatic representation of the model is given below: 


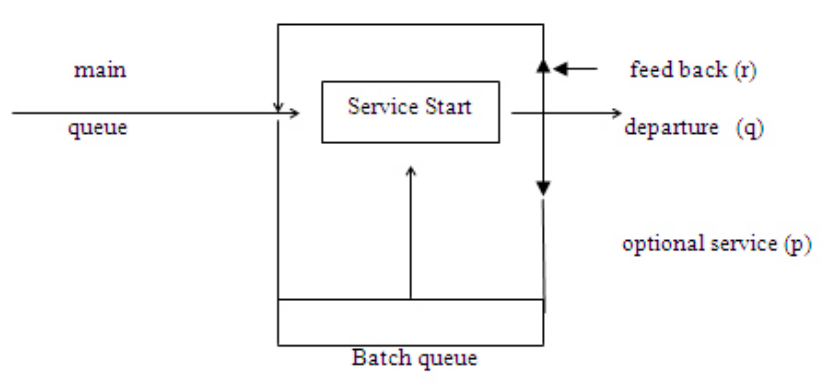

\section{The Queue length process}

Let $Y_{n}=u(u=1,2)$ be the type of service completion at the $\mathrm{n}^{\text {th }}$ out put epoch, defined as follows:

$$
Y_{n}=\left\{\begin{array}{l}
1, \text { if the } n^{\text {th }} \text { output seeks essential service } \\
2, \text { if the } n^{\text {th }} \text { output seeks optional service }
\end{array}\right.
$$

and $M_{\ell}, N_{\ell}$ are respectively the number in the main queue and the number of the batch queue of the system immediately after the $\ell^{\text {th }}$ output. The sequence ( $M_{\ell}, N_{\ell}$ ) is called the embedded queue length process at the $\ell^{\text {th }}$ output epoch and is the process of interest here. The state space is $\{0,1, \ldots\} \times\{0,1, \ldots, b\}$.

In the steady state, the joint probabilities are

$$
\mathrm{P}_{\mathrm{m}, \mathrm{n}}^{(\mathrm{i})}=\mathrm{P}_{\mathrm{r}}\left\{M_{\ell}=\mathrm{m}, N_{\ell}=\mathrm{n}\right\}
$$

where $i=1$, means the server is busy with the main queue and $i=2$, implies that the server is busy with a batch.

And $\mathrm{Q}=\operatorname{Pr}\{$ the server is idle $\}$

Consequently, the set of statistical equilibrium equations for the probabilities in (3.1) and (3.2) are as follows ;

$$
\begin{aligned}
& \left(\lambda+\mu_{1}\right) \mathrm{P}_{\mathrm{m}, \mathrm{n}}^{(1)}=\lambda \mathrm{P}_{\mathrm{m}-1, \mathrm{n}}^{(1)}+r \mu_{1} \mathrm{P}_{\mathrm{m}-1, \mathrm{n}}^{(1)}+ \\
& q \mu_{1} \mathrm{P}_{\mathrm{m}+1, \mathrm{n}}^{(1)}+p \mu_{1} \mathrm{P}_{\mathrm{m}+1, \mathrm{n}}^{(1)}: \mathrm{m}>0,0<\mathrm{n} \leq \mathrm{b}
\end{aligned}
$$

$$
\left(\lambda+\mu_{1}\right) \mathrm{P}_{\mathrm{m}, 0}^{(1)}=q \mu_{1} \mathrm{P}_{\mathrm{m}+1,0}^{(1)}+\lambda \mathrm{P}_{\mathrm{m}-1,0}^{(1)}+\mu_{2}
$$$$
\mathrm{P}_{\mathrm{m}+1,0}^{(2)}: \mathrm{m}>0
$$

$\left(\lambda+\mu_{1}\right) \mathrm{P}_{0, \mathrm{n}}^{(1)}=p \mu_{1} \mathrm{P}_{1, \mathrm{n}-1}^{(1)}+q \mu_{1} \mathrm{P}_{1, \mathrm{n}}^{(1)}: 0<\mathrm{n}<\mathrm{b}$

$$
\left(\lambda+\mu_{1}\right) \mathrm{P}_{0,0}^{(1)}=\lambda \mathrm{Q}+q \mu_{1} \mathrm{P}_{1,0}^{(1)}+\mu_{2} \mathrm{P}_{1,0}^{(2)}
$$

$$
\begin{aligned}
& \left(\lambda+\mu_{2}+r \mu_{1}\right) \mathrm{P}_{\mathrm{m}, \mathrm{b}}^{(2)}=\lambda \mathrm{P}_{\mathrm{m}-1,0}^{(2)}+p \mu_{1} \mathrm{P}_{\mathrm{m}, \mathrm{b}-1}^{(1)}: \mathrm{m}>0 \\
& \left(\lambda+\mu_{2}\right) \mathrm{P}_{0,0}^{(2)}=p \mu_{1} \mathrm{P}_{0, \mathrm{n}-1}^{(1)}: 0<\mathrm{n} \leq \mathrm{b} \\
& \lambda \mathrm{Q}=q \mu_{1} \mathrm{P}_{0,0}^{(1)}+\mu_{2} \mathrm{P}_{0,0}^{(2)}+r \mu_{1} \mathrm{P}_{0,0}^{(1)}
\end{aligned}
$$

We can solve the above equations through the following partial generating functions

$$
\begin{aligned}
& \mathrm{P}^{(1)}(\alpha)=\sum_{m=0}^{\infty} P^{(1)}{ }_{m, n} \alpha^{m} \\
& \mathrm{P}^{(1)}(\beta)=\sum_{n=0}^{\infty} P_{m, n}^{(1)} \beta^{n} \\
& \mathrm{P}^{(1)}(\alpha, \beta)=\sum_{m=0}^{\infty} \sum_{n=0}^{\infty} \mathrm{P}_{\mathrm{m}, \mathrm{n}}^{(1)} \alpha^{\mathrm{m}} \beta^{\mathrm{n}}
\end{aligned}
$$

$\mathrm{P}_{0}^{(2)}(\alpha)=\sum_{m=0}^{\infty} P_{m, 0}^{(2)} \alpha^{m}$

Use of (3.10) - (3.13) in (3.3) - (3.9), yields the following equations:

$\left[\left(\lambda(1-\alpha)+\mu_{1}-r \mu_{1} \alpha\right) \alpha-q \mu_{1}\right] \mathrm{P}^{(1)}{ }_{\mathrm{n}}(\alpha)=p \mu_{1} \mathrm{P}^{(1)}{ }_{\mathrm{n}-1}$
$(\alpha)-p \mu_{1} \mathrm{P}^{(1)}{ }_{0, \mathrm{n}-1}-q \mu_{1} \mathrm{P}_{0, \mathrm{n}}^{(1)} ; 0<\mathrm{n} \leq \mathrm{b}-1$

$\left[\left(\lambda(1-\alpha)+\mu_{1}\right) \alpha-q \mu_{1}\right] \mathrm{P}^{(1)}{ }_{0}(\alpha)=\lambda \alpha Q-\mu_{2} \mathrm{P}_{0,0}^{(2)}$

- $q \mu_{1} \mathrm{P}_{0,0}^{(1)}+\mu_{2} \mathrm{P}_{0}^{(2)}(\alpha)$

$\left[\lambda(1-\alpha)+\mu_{2}+r \mu_{1}\right] \mathrm{P}_{0}^{(2)}(\alpha)=p \mu_{1} \mathrm{P}_{\mathrm{b}-1}^{(1)}(\alpha)+$ $r \mu_{1} \mathrm{P}_{0,0}^{(2)}$

Multiplying equation (3.14) by $\beta^{\mathrm{n}}$ and summing over $\mathrm{n}$, we get

$P^{(1)}(\alpha, \beta)=$

$\frac{\lambda \alpha Q+\mu_{2} P^{(2)}{ }_{0}(\alpha)-\mu_{1}(p \beta+q) P^{(1)}{ }_{0}(\beta)-\mu_{2} P^{(2)}{ }_{0,0}}{\left[\lambda(1-\alpha)+\mu_{1}\right] \alpha-\mu_{1}(p \beta+r \alpha+q)}+$

$\frac{\mu_{1}(r \alpha-p \beta) P^{(1)}{ }_{0}(\alpha)+p \mu_{1} P^{(1)}{ }_{0, b-1} \beta^{b+1}}{\left[\lambda(1-\alpha)+\mu_{1}\right] \alpha-\mu_{1}(p \beta+r \alpha+q)}$

In (3.8), $\mathrm{n}=1$ (3.8) yields, 
$\mathrm{P}_{0,0}^{(2)}=\frac{p \mu_{1}}{\lambda+\mu_{2}} \mathrm{P}_{0,0}^{(1)}$

Using this in (3.9), we get

$Q=\frac{\mu_{1}(q+r)\left(\lambda+\mu_{2}\right)+p \mu_{1} \mu_{2}}{\lambda\left(\lambda+\mu_{2}\right)} \mathrm{P}_{0,0}^{(1)}$

$\mathrm{P}^{(1)}{ }_{0,0}=\frac{\lambda\left(\lambda+\mu_{2}\right) Q}{\mu_{1}(q+r)\left(\lambda+\mu_{2}\right)+p \mu_{1} \mu_{2}}$

From (3.8) we've

$\mathrm{P}_{0,0}^{(2)}=\frac{\lambda r \mu_{1} Q}{\mu_{1}(q+r)\left(\lambda+\mu_{2}\right)+p \mu_{1} \mu_{2}}$

Multiplying equation (3.8) by $\beta^{n}$ and summing from $\mathrm{n}=1$ to $b$, we get

$\mathrm{P}_{0}^{(1)}(\beta)=\frac{\lambda\left(\lambda+\mu_{2}\right)\left(1-\beta^{b}\right) Q}{\left[\mu_{1}(q+r)\left(\lambda+\mu_{2}\right)+p \mu_{1} \mu_{2}\right](1-\beta)}$

In equation (3.17) replacing the value of $\mathrm{P}^{(2)}{ }_{(0,0)}$ from (3.19) and that of $\mathrm{P}^{(1)}{ }_{0}(\beta)$ from (3.20), we get

$P^{(1)}(\alpha, \beta)=\frac{A}{B}$

where

$A=\left\{(1-\beta)\left[\lambda(1-\alpha)+\mu_{2}+r \mu_{1}\right]\left[\left(\mu_{1}(q+r)\left(\lambda+\mu_{2}\right)+\right.\right.\right.$ $\left.p \mu_{1} \mu_{2}\right) \lambda \alpha-p \lambda \mu_{1} \mu_{2}+p \lambda \mu_{1} \beta^{b+1}$

$\left.\left(\lambda+\mu_{2}\right)\right] \lambda \mu_{1}(q+p \beta)\left(1-\beta^{b}\right)\left(\lambda+\mu_{2}\right)[\lambda(1-\alpha)+$

$\left.\left.\mu_{2}+r \mu_{1}\right]+\lambda \mu_{1}^{2} \mu_{2 p}(1-\beta) r\right\} Q$

$+\left\{(1-\beta)\left[(q+r) \mu_{1}\left(\lambda+\mu_{2}\right)+p \mu_{1} \mu_{2}\right]\left(\mu_{1}(r \alpha-p \beta)\right.\right.$

$\left.\left.\left[\lambda(1-\alpha)+\mu_{2}+r \mu_{1}\right]+p \mu_{1} \mu_{2}\right)\right\} P^{(1)}(\alpha)$

and

$B=\left[\left(\lambda(1-\alpha)+\mu_{1}\right) \alpha-\mu_{1}(q+r \alpha+p \beta)\right]\left[(q+r) \mu_{1}(\lambda+\right.$ $\left.\left.\mu_{2}\right)+p \mu_{1} \mu_{2}\right](1-\beta)\left[\lambda(1-\alpha)+\mu_{2}+r \mu_{1}\right]$

Now we note that for $\beta=0, \mathrm{P}^{(1)}(\alpha, \beta)=\mathrm{P}^{(1)}(\alpha)$.

Thus for $\beta=0$, equation (3.21) becomes
$\mathrm{P}^{(1)}{ }_{0}(\alpha)=\frac{C Q}{D}$

where

$D=\left[\mu_{1}(q+r)\left(\lambda+\mu_{2}\right)+p \mu_{1} \mu_{2}\right]\{[(\lambda(1-\alpha)+$

$\left.\left.\mu_{1}\right) \alpha-\mu_{1}(q+r \alpha)\right]\left[\lambda(1-\alpha)+\mu_{2}+r \mu_{1}\right]$

$\left.-\left(\mu_{1} r \alpha\left[\lambda(1-\alpha)+\mu_{2}+r \mu_{1}\right]+p \mu_{1} \mu_{2}\right)\right\}$

In equation (3.16) $b=1$ gives,

$\mathrm{P}^{(2)}{ }_{0}(\alpha)=\frac{\mu_{1} p P^{(1)}{ }_{0}(\alpha)+r \mu_{1} P^{(2)}{ }_{00}}{\lambda(1-\alpha)+\mu_{2}+r \mu_{1}}$

using (3.22) in (3.23)

$\mathrm{P}_{0}^{(2)}(\alpha)=\frac{E Q}{F}$

where

$E=p \mu_{1}\left[\left(\lambda(1-\alpha)+\mu_{2}+r \mu_{1}\right)\left(\left[(q+r) \mu_{1}\left(\lambda+\mu_{2}\right)+\right.\right.\right.$

$\left.\left.p \mu_{1} \mu_{2}\right] \lambda \alpha-p \lambda \mu_{1} \mu_{2}\right)-\lambda \mu_{1} q\left(\lambda+\mu_{2}\right)(\lambda(1-\alpha)$

$\left.\left.+\mu_{2}+r \mu_{1}\right)+\lambda \mu_{1}^{2} \mu_{2} r p\right]+\lambda \mu_{1}^{2} p r\left[\left(\left(\lambda(1-\alpha)+\mu_{1}\right) \alpha-\right.\right.$

$\left.\mu_{1}(q+r \alpha)\right)\left(\lambda(1-\alpha)+\mu_{2}+r \mu_{1}\right)$

$\left.-\left(r \mu_{1} \alpha\left(\lambda(1-\alpha)+\mu_{2}+r \mu_{1}\right)+p \mu_{1} \mu_{2}\right)\right]$

$F=\left[\lambda(1-\alpha)+\mu_{2}+r \mu_{1}\right]\left[\mu_{1}(p+r)\left(\lambda+\mu_{2}\right)+\right.$ $\left.p \mu_{1} \mu_{2}\right]\left\{\left[\lambda(1-\alpha)+\mu_{1}\right) \alpha-\mu_{1}(q+r \alpha)\right][\lambda(1-\alpha)+$ $\left.\left.\mu_{2}+r \mu_{1}\right]\left[r \alpha \mu_{1}\left(\lambda(1-\alpha)+\mu_{2}+r \mu_{1}\right)+p \mu_{1} \mu_{2}\right]\right\}$

The normalizing condition is

$\mathrm{P}^{(1)}{ }_{0}(1)+\mathrm{P}^{(2)}{ }_{0}(1)+\mathrm{Q}=1$

Using (3.22),(3.24) and (3.25), we have

$\mathrm{Q}=\frac{H I}{(G+H) I+J}$

where

$G=\left(r \mu_{1}+\mu_{2}\right)\left\{\left[\mu_{1}(q+r)\left(\lambda+\mu_{2}\right)+\right.\right.$

$\left.\left.p \mu_{1} \mu_{2}\right] \lambda-p \lambda \mu_{1} \mu_{2}\right\}-\lambda \mu_{1} q(\lambda+$

$\left.\mu_{2}\right)\left(r \mu_{1}+\mu_{2}\right)+\lambda \mu_{1}{ }^{2} \mu_{2} r p$ 


$$
\begin{aligned}
& H=\mu_{1} p\left\{\left[\mu_{1}(q+r)\left(\lambda+\mu_{2}\right)+p \mu_{1} \mu_{2}\right]\right. \\
& \left(r \mu_{1}+\mu_{2}\right)-\left[\mu_{1}(q+r)\left(\lambda+\mu_{2}\right)+p \mu_{1} \mu_{2}\right] \\
& -\left(\mu_{1} r\left(r \mu_{1}+\mu_{2}\right)+p \mu_{1} \mu_{2}\right) \\
& I=r \mu_{1}+\mu_{2} \\
& J=\mu_{1} p\left[\left(\left[\mu_{1}(p+r)\left(\lambda+\mu_{2}\right)+p \mu_{1} \mu_{2}\right] \lambda-\right.\right. \\
& \left.\left.\lambda p \mu_{1} \mu_{2}\right)\left(r \mu_{1}+\mu_{2}\right)\right]-\lambda \mu_{1}\left(\lambda+\mu_{2}\right)\left(r \mu_{1}+\mu_{2}\right) \\
& \left.+\lambda \mu_{1}^{2} \mu_{2} p r\right]+\lambda \mu_{1}^{2} p r\left[\mu_{1} p\left(r \mu_{1}+\mu_{2}\right)-\right. \\
& \left.\left(\mu_{1} r\left(r \mu_{1}+\mu_{2}\right)+p \mu_{1} \mu_{2}\right)\right]
\end{aligned}
$$

$\mathrm{Q}$ in (3.26) together with (3.22) and (3.24) yields the required probability generating functions.

\section{The Moments of Queue Length Process}

Let $\mathrm{L}_{\mathrm{q}}{ }^{(1)}$ and $\mathrm{L}_{\mathrm{q}}{ }^{(2)}$ denote the average queue length of the waiting customers when the server is busy in the main queue and batch queue respectively. Then

$$
\mathrm{L}_{q}^{(i)}=\sum_{m=0}^{\infty} m P_{m}^{(i)}=\left[\frac{d}{d \alpha} P^{(i)}(\alpha)\right]_{\alpha=1} ; \mathrm{i}=1,2
$$

Applying suitable manipulation on (3.24) and (3.25) with (3.27) we have

$$
\mathrm{L}_{\mathrm{q}}^{(1)}=\frac{D_{1}\left[N_{1}^{\prime} Q+N_{1} Q^{\prime}\right]-N_{1} Q D_{1}^{\prime}}{D_{1}^{2}}
$$

where

$$
\begin{aligned}
& N_{1}=\lambda \mu_{1} r\left[\left(\lambda+\mu_{2}\right)\left(r \mu_{1}+\mu_{2}\right)+\mu_{1} \mu_{2} p\right] \\
& D_{1}=\mu^{2}{ }_{1}\left[(q+r)\left(\lambda+\mu_{2}\right)+p \mu_{2}\right] \\
& {\left[(1-p)\left(\mu_{2}+r \mu_{1}\right)-2 r\left(\mu_{2}+r \mu_{1}\right)-p \mu_{2}\right]} \\
& N_{1}^{\prime}=\lambda\left(\mu_{2}+r \mu_{1}\right)\left[(q+r) \mu_{1}\left(\lambda+\mu_{2}\right)+p \mu_{1} \mu_{2}\right]- \\
& \lambda^{2}\left[(q+r) \mu_{1}\left(\lambda+\mu_{2}\right)-p \mu_{1} \mu_{2}\right]+\lambda^{2} \mu_{1} q\left(\lambda+\mu_{2}\right) \\
& D_{1}^{\prime}=\left(\mu_{1}-\lambda-\mu_{1} r\right)\left(r \mu_{1}+\mu_{2}\right)\left[\left(q \mu_{1}+r \mu_{1}\right)\left(\lambda+\mu_{2}\right)+p \mu_{1} \mu_{2}\right]- \\
& \lambda\left[\mu_{1}-\mu_{1}(q+r)\right]\left[(q+r) \mu_{1}\left(\lambda+\mu_{2}\right)+\mu_{1} \mu_{2}\right]-\left[(q+r) \mu_{1}(\lambda+\right. \\
& \left.\left.\mu_{2}\right)+\mu_{1} \mu_{2}\right]\left[-\lambda \mu_{1} r+\mu_{1} r\left(\mu_{2}+r \mu_{1}\right)\right] \\
& Q^{\prime}=0
\end{aligned}
$$$$
\mathrm{L}_{\mathrm{q}}^{(2)}=\frac{D_{2}\left[N_{2}^{\prime} Q+N_{2} Q^{\prime}\right]-N_{2} Q D_{2}^{\prime}}{D_{2}^{2}}
$$

where

$$
\begin{aligned}
& N_{2}=p \mu_{1}\left[\lambda ( \mu _ { 2 } + r \mu _ { 1 } ) \left(\left[(q+r) \mu_{1}\left(\lambda+\mu_{2}\right)+\mu_{1} \mu_{2} p\right]-\right.\right. \\
& \left.\mu_{1} \mu_{2} p\right)-\lambda \mu_{1} q\left(\lambda+\mu_{2}\right)\left(\mu_{2}+r \mu_{1}\right) \\
& \left.+\lambda \mu_{1}^{2} \mu_{2} r p\right]+\lambda \mu_{1}^{2} r p\left[\left(\mu_{1}-(q+r) \mu_{1}\left(\mu_{2}+r \mu_{1}\right)-\right.\right. \\
& \left.\left(r \mu_{1}\left(\mu_{2}+r \mu_{1}\right)+\mu_{1} \mu_{2} p\right)\right] \\
& D_{2}=\left(\mu_{2}+r \mu_{1}\right)\left[\left(\lambda+\mu_{2}\right) \mu_{1}(q+r)+p \mu_{1} \mu_{2}\right] \\
& {\left[\left(\mu_{1}-\mu_{1}(q+r)\right)\left(r \mu_{1}+\mu_{2}\right)-\left(\mu_{1} r\left(\mu_{2}+r \mu_{1}\right)\right.\right.} \\
& \left.\left.+\mu_{1} \mu_{2} p\right)\right] \\
& N_{2}^{\prime}=p \mu_{1}\left[-\lambda\left(\left[(q+r) \mu_{1}\left(\lambda+\mu_{2}\right)+p \mu_{1} \mu_{2}\right] \lambda-\right.\right. \\
& \left.\lambda p \mu_{1} \mu_{2}\right)+\left(r \mu_{1}+\mu_{2}\right) \lambda\left[(q+r) \mu_{1}\left(\lambda+\mu_{2}\right)\right. \\
& \left.\left.+p \mu_{2} \mu_{1}\right]+\lambda^{2} \mu_{1} q\left(\lambda+\mu_{2}\right)\right]+\lambda \mu_{1}^{2} r p\left[\left(\mu_{1}-\lambda-\right.\right. \\
& \left.r \mu_{1}\right)\left(\mu_{2}+r \mu_{1}\right)-\lambda\left(\mu_{1}-\mu_{1}(q+r)\right. \\
& \left.-r \mu_{1}\left(\mu_{2}+r \mu_{1}\right)+r \lambda \mu_{1}\right] \\
& D_{2}^{\prime}=-\lambda\left[(q+r) \mu_{1}\left(\lambda+\mu_{2}\right)+p \mu_{1} \mu_{2}\right]\left[\left(\mu_{1}-\mu_{1}(q+\right.\right. \\
& \left.r))\left(\mu_{2}+r \mu_{1}\right)-r \mu_{1}\left(r \mu_{1}+\mu_{2}\right)-p \mu_{2} \mu_{1}\right]+\left(\mu_{2}+r \mu_{1}\right) \\
& {\left[(q+r) \mu_{1}\left(\lambda+\mu_{2}\right)+p \mu_{1} \mu_{2}\right]\left[( \mu _ { 1 } - \lambda - r \mu _ { 1 } ) \left(\mu_{2}+\right.\right.} \\
& \left.\left.r \mu_{1}\right)-\lambda\left(\mu_{1}-\mu_{1}(q+r)\right)-r \mu_{1}\left(\mu_{2}+r \mu_{1}\right)+r \lambda \mu_{1}\right]
\end{aligned}
$$

and $\mathrm{L}_{\mathrm{q}}$, the mean number of customers in the queue irrespective of type of queue (main queue or batch queue) is

$\mathrm{L}_{\mathrm{q}}=\mathrm{L}_{\mathrm{q}}^{(1)}+\mathrm{L}_{\mathrm{q}}{ }^{(2)}$

By -Little's formula, the mean waiting time in the queue is

$$
\mathrm{W}_{\mathrm{q}}=\mathrm{L}_{\mathrm{q}} / \lambda
$$

\section{Particular Case}

Case (i): On setting $r=0$ (no feedback) (3.22), (3.24) and (3.26) become,

$$
\mathrm{P}_{0}^{(1)}(\alpha)=\frac{\lambda\left(\lambda(1-\alpha)+\mu_{2}\right) Q}{\lambda^{2} \alpha^{2}-\lambda\left(\lambda+\mu_{1}+\mu_{2}\right) \alpha+\mu_{1}\left(\lambda q+\mu_{2}\right)}
$$

$\mathrm{P}_{0}^{(2)}(\alpha)=\frac{\lambda \mu_{1} p Q}{\lambda^{2} \alpha^{2}-\lambda\left(\lambda+\mu_{1}+\mu_{2}\right) \alpha+\mu_{1}\left(\lambda q+\mu_{2}\right)}$

and

$$
\mathrm{Q}=1-\frac{\lambda}{\mu_{1}}\left[1+\frac{p \mu_{1}}{\mu_{2}}\right]
$$


These equations coincide with the equations (21), (22) and (24) of Madan (1992).

Case (ii): On setting $\mathrm{p}=0$ (no optional service) (3.22), (3.24) and (3.26) become,

$\mathrm{P}_{0}^{(1)}(\alpha)=\frac{\lambda \alpha Q}{\left[\lambda(1-\alpha)+\mu_{1}\right] \alpha+r \mu_{1}}, \quad \mathrm{P}_{0}^{(2)}(\alpha)=0$

$\mathrm{Q}=\frac{\mu_{1}(1+r)}{\lambda+\mu_{1}(1+r)}$

These results are the well-known results of $\mathrm{M} / \mathrm{M} / 1 / \infty$ feedback queue except for notation.

Case (iii): $\mathrm{p}=0, \mathrm{r}=0$ (no feedback and no optional service) (3.22), (3.24) and (3.26) gives well known results for $\mathrm{M} / \mathrm{M} / 1$ queue except for notation

$\mathrm{P}_{0}^{(1)}(\alpha)=\frac{\lambda \alpha Q}{\left[\lambda(1-\alpha)+\mu_{1}\right] \alpha}$

$\mathrm{P}^{(2)}{ }_{0}(\alpha)=0$

$\mathrm{Q}=1-\frac{\lambda}{\mu_{1}}$

\section{Numerical study}

In this section we analyze the operating characteristics i.e, the queue length $\left(\mathrm{L}_{\mathrm{q}}\right)$ and queue waiting time $\left(\mathrm{W}_{\mathrm{q}}\right)$ of our model by assigning suitable numerical values to the parameters under study. First we fix the values of $\mu_{1}, \mu_{2,}$ p and $\mathrm{r}$. The values of $\mathrm{L}_{\mathrm{q}}$ and $\mathrm{W}_{\mathrm{q}}$ are obtained using the formulae derived in section 4 and are shown in tables 6.1 and 6.2.

For the analysis we take $\mu_{1}=0.7, \mu_{2}=0.7, p=0.5$, and $\mathrm{r}=0.4$, the measures $\mathrm{L}_{\mathrm{q}}$ and $\mathrm{W}_{\mathrm{q}}$ are calculated using the formulae in (4.4) and (4.5). Also as a measure of comparison we calculate the $\mathrm{L}_{\mathrm{q}}$ and $\mathrm{W}_{\mathrm{q}}$ for the system without feedback ( $r=0$, called model-II ), for the system without optional service ( $p=0$, called model-III) and for the system both without feedback and optional service ( $r=$ $\mathrm{p}=0$, called model-IV). We denote the system in this paper as model-I. The calculated mean queue lengths $\mathrm{L}_{\mathrm{q}}$ for various values of $\lambda$ are given in the table 6.1 and the corresponding mean waiting times $\mathrm{W}_{\mathrm{q}}$ are given in table 6.2 .
In table 6.1, the mean queue length for different special cases related to the model studied in this paper is presented along with that of model-I. It can be observed that the mean queue length for the system with feedback and second optional service (Model-I) is increasing rapidly with the arrival rate when compared with the other cases as expected. This is due to the flexibility of the server to provide more number of services to the customer for the asking.

Table 6.1: Mean queue length

\begin{tabular}{|c|c|c|c|c|}
\hline & Model-I & Model-II & Model-III & Model-IV \\
\hline 0.1 & 0.57 & 0.44 & 0.44 & 0.24 \\
\hline 0.2 & 1.34 & 1.19 & 0.94 & 0.57 \\
\hline 0.3 & 2.40 & 2.84 & 1.58 & 1.07 \\
\hline 0.4 & 3.89 & 9.37 & 2.38 & 1.90 \\
\hline 0.5 & 6.06 & ----- & 3.38 & 3.57 \\
\hline 0.6 & 9.45 & ----- & 4.64 & 8.57 \\
\hline 0.7 & 15.26 & ----- & 6.27 & ---- \\
\hline 0.8 & 27.17 & ----- & 8.43 & ----- \\
\hline 0.9 & 63.57 & ----- & 11.37 & ----- \\
\hline 1.0 & ----- & ----- & 15.54 & ----- \\
\hline
\end{tabular}

In table 6.2, the mean waiting time for different special cases related to the model studied in this paper is presented along with that of model-I. It can be observed that the mean waiting time for the system with feedback and second optional service (Model-I) is increasing rapidly with the arrival rate when compared with the other cases as expected. This is also again due to the flexibility of the server to provide more time period of service given to the customers, those who ask.

Table 6.2: Mean waiting time

\begin{tabular}{|c|c|c|c|c|}
\hline$\lambda$ & Model-I & Model-II & Model-III & Model-IV \\
\hline 0.1 & 0.06 & 0.04 & 0.04 & 0.02 \\
\hline 0.2 & 0.27 & 0.24 & 0.19 & 0.11 \\
\hline 0.3 & 0.72 & 0.85 & 0.48 & 0.32 \\
\hline 0.4 & 1.56 & 0.75 & 0.95 & 0.76 \\
\hline 0.5 & 3.03 & ------ & 1.69 & 1.79 \\
\hline 0.6 & 5.67 & ----- & 2.78 & 5.14 \\
\hline 0.7 & 10.69 & ------ & 4.39 & ----- \\
\hline 0.8 & 21.73 & ----- & 6.74 & ----- \\
\hline 0.9 & 57.21 & ----- & 10.23 & ----- \\
\hline 1.0 & ------ & ----- & 15.54 & ----- \\
\hline
\end{tabular}

\section{Conculsion}

In this paper we analyze a simple $\mathrm{M} / \mathrm{M} / 1$ feed back queueing system where the server provides two type of 
services, an essential service to all the customers and an additional optional batch service. For this model the probability generating function in steady state has been obtained. Some performance measures related to the queueing system are calculated. Particular cases and numerical examples are also presented to test integrity of the model. Our computational study shows the difference between the existing one and the model in this paper.

\section{ACKNOWLEDGEMENT}

The authors would like to thank the anonymous referee for his comments which helped to improve the quality and clarity of the paper.

\section{REFERENCES}

[1] G.R. D’ Avignon and R.L. Disney, Management Sciences, Vol.24, No. 2, (1976)168 - 180.

[2] R. Kalyanaraman, N. Thillai Govindan and G. Ayyappan, Octogon, Vol.10, No.2, (2002)583-591.

[3] L. Kleinrock, Queuing Systems, Vol.I : Theory, Wiley, New York, 1975.

[4] K.C. Madan, J.Appl. Prob., Vol.12, (1975)195-200.

[5] K.C. Madan, IAPQR Transactions, Vol. 17, No.1, 1992.

[6] L. Takacs, Bell Systems Technical Journal, Vol.42, (1963)509-519. 